\title{
El diálogo y el plurilingüismo en el aula
}

\author{
Dora Riestra \\ Universidad Nacional de Río Negro, Argentina \\ dora.riestra@gmail.com
}

\begin{abstract}
Resumen: Uno de los desafíos que tenemos en el contexto de la educación básica obligatoria, en la enseñanza de lenguas, en América del sur es reconceptualizar el diálogo en el aula frente al plurilingüismo en sus diversas manifestaciones culturales actuales. Estamos investigando el habla dialogal desde la perspectiva de Jakubinskij (2018) y el concepto de lengua funcional de Coseriu (1992) con la finalidad de aportar a la práctica profesional docente en enseñanza de lenguas. La metodología que desarrollamos apunta a aportar instrumentos de evaluación para la práctica profesional docente, en función de la interacción discursivo-textual en el aula.
\end{abstract}

Palabras clave: Diálogo- Habla dialogal- Plurilingüismo-Lenguas funcionales-Interacción discursivo-textual.

Recibido: 28/04/2019

Aceptado: 06/07/2019 


\title{
Dialogue and plurilingualism in the classroom
}

\begin{abstract}
One of the challenges we have in the context of basic education, in languages teaching, in South America is to reconceptualize classroom dialogue in the face of plurilingualism in its diverse current cultural manifestations. We are investigating the dialogic speech from the perspective of Jakubinskij (2018) and the concept of functional language of Coseriu (1992) with the purpose of contributing to the professional teaching practice in language teaching. The methodology we develop aims to provide assessment tools for professional teaching practice, based on the discursive-textual interaction in the classroom.
\end{abstract}

Keywords: Dialogue - Dialogue Speech - Plurilingualism - Functional Languages Discursive-textual interaction.

\section{Diálogo e pluriliguismo em sala de aula}

Resumo: Um dos desafios que temos no contexto da educação básica obrigatória, na educação de idiomas, na América do Sul é o de reconceitualizar o diálogo em sala de aula em face do plurilinguismo em suas diversas manifestaçôes culturais atuais. Estamos investigando a fala dialogal a partir da perspectiva de Jakubinskij (2018) e do conceito de lingua funcional de Coseriu (1992) com o propósito de contribuir para a prática profissional docente no ensino de línguas. A metodologia que desenvolvemos tem como objetivo fornecer ferramentas de auto-avaliação para a prática docente profissional, com base na interação discursivo-textual em sala de aula.

Palavras-chave: Diálogo - Fala dialogal - Plurilinguismo - Linguas Funcionais- Interação discursivo-textual. 


\section{Por qué reconceptualizar el diálogo en la escuela}

La investigación que estamos comenzando se basa en las que hemos realizado anteriormente en didáctica de la lengua materna, que ahora extendemos a las lenguas en plural. La falta de enseñanza de la gramaticalidad de la lengua materna es un problema que fue acentuándose a medida en que fueron introduciéndose los marcos teóricos de la lingüística en primer término y posteriormente, de la lingüística sistémico-funcional. De esta manera, en las clases de lengua de nuestro país se sustituyó la llamada descripción o análisis gramatical por la descripción discursivo-textual.

Debido a que las nociones gramaticales como objeto de enseñanza fueron desapareciendo, por las sustituciones mencionadas, las clases de lengua no tienen el efecto de enseñanza- aprendizaje esperado en el desarrollo de las actividades de lectura y escritura de nuestros alumnos.

Esta es una problemática reconocida por los profesores y los didactas, y hemos dedicado varias investigaciones desde el interaccionismo sociodiscursivo en nuestro equipo de trabajo, como lo expondremos más delante. Nuestro objeto de investigación estaba centrado en la transposición didáctica para la enseñanza de las lenguas en general -y en particular el castellano-, en los niveles primario y secundario en el contexto argentino.

En la aplicación de la propuesta didáctica, planteada y elaborada en la formación de profesores de lengua, los límites metodológicos aparecieron en el momento de la instrumentación en el aula que, en principio, fue relevado como un problema de la comunicación que impedía transmitir las nociones técnicas de la asignatura.

Las traducciones de Jakubinskij $(2014 ; 2018)$ nos permitieron abrir una línea de investigación nueva para abordar el diálogo de las clases y, en esta investigación que comenzamos, apuntamos a elaborar instrumentos de autoevaluación de la propia tarea de enseñar las lenguas y sus gramáticas particulares, incorporando el análisis del diálogo. Desarrollaremos aquí el marco teórico y el diseño metodológico, puesto que aún no contamos con una proporción de los análisis realizados que nos permitan mostrar algunos resultados.

\section{El concepto de plurilingüismo}

Abordaré dos dimensiones del concepto de plurilingüismo para delimitar su alcance y, sobre todo, para explicar el sentido que le atribuyo en los contextos de enseñanza de las lenguas. En primer lugar, la problemática de la diversidad de lenguas y su carácter cultural fue abordada por Humboldt (1767-1835), quien se refirió a la lengua general y a la lengua singular, esta última entendida como habla cotidiana banal (Humboldt, 1991, p.43). Dicha tradición no fue tomada por la mayoría de los lingüistas posteriores, con algunas excepciones en las que nos referenciamos (Saussure, Jakubinskij, Voloshinov, Coseriu). Se trata de una dimensión del plurilingüismo como situación humana, un tema interesante, aunque aquí lo tomaré apenas como fundamento histórico-conceptual. El reconocimiento de las lenguas como producciones culturales es un descubrimiento que hace Wilhelm von Humboldt utilizando los datos acopiados por su hermano Alexander, que le permitieron comparar lenguas diversas y entenderlas histórica y geográficamente, en diferentes regiones del planeta.

Uno de los lingüistas del siglo XX que conoció las investigaciones humboldtianas fue el propio Saussure (2004), quien lo cita en sus escritos. Con posterioridad, en los años cincuenta del siglo XX, también Coseriu (1992) analizó la relación entre el lenguaje y las lenguas al diferenciar las lenguas históricas y las lenguas funcionales, ya que son estas las que realizan las primeras; en la obra coseriana la referencia de Humboldt está presente en esta misma dirección de la pluralidad de las lenguas. 
Por otra parte, Rastier (2012) distingue las lenguas de comunicación y de cultura de las lenguas de servicio. Las primeras, las lenguas de cultura, son las que en su aprendizaje conllevan la intencionalidad de comprender otros pueblos con sus bagajes culturales. Las segundas son básicamente las que se utilizan como lenguas que sirven para una finalidad con una concepción instrumental de la comunicación; sería en la actualidad el caso del inglés que se usa en aeropuertos y transacciones comerciales por internet, entre otras situaciones comunicativas. En consecuencia, la distinción de Rastier muestra que se trataría de dos actitudes ante la incorporación de una segunda lengua.

El concepto europeo de plurilingüismo surgió en los años noventa, en defensa de las lenguas europeas que fueron hegemónicas en algún tiempo y hoy están siendo solapadas por el inglés como lengua de comunicación. Según el mismo Rastier (2012), se trataría de una lengua de servicio.

Observamos que la tradición plurilingüista se basa en el uso de las lenguas, mientras en Europa la tradición también llamada multilingüista reconoce la existencia de muchas lenguas, como lo hiciera la escuela de los neogramáticos del siglo XIX (que se continúa en muchas tendencias teóricas actuales). Esta última se basa en la descripción y la comparación de las formas las lenguas, lo que constituye un punto de partida o enfoque diferente, que podríamos identificar como técnico o tecnicista como tendencia.

Por lo tanto, encontramos aquí dos hilos conductores para analizar las lenguas: el descriptivismo y el uso, dos dimensiones de análisis que llegan a la escuela aún hoy.

Para nosotros, latinoamericanos, el plurilingüismo es, además, otro fenómeno o, según lo definamos, se produce en una situación política diferente de la de Europa. Somos plurilingües por diversos orígenes socio-históricos: 1) las lenguas de los pueblos originarios que están en todo el territorio latinoamericano aun cuando muchas de ellas se encuentren relegadas por la hegemonía de las lenguas europeas de los colonizadores; 2) están las lenguas de la colonización, que abarcan más que el castellano y el portugués oficiales, ya que se mezclaron con lenguas eslavas, con el italiano, el francés, el alemán, el polaco, etc., de los inmigrantes de los siglos XIX y XX. Como ejemplos de esto, en Paraguay está institucionalizado el bilingüismo y en Bolivia la plurinacionalidad, que conlleva el plurilingüismo en su concepción.

Por todo esto que apuntamos, acordamos teóricamente con Coseriu (1992) respecto de la relación de la lengua histórica con las lenguas funcionales (las que realmente funcionan social y regionalmente); en este sentido, el castellano, considerado como lengua histórica, se realiza en diversas lenguas funcionales en el continente americano, tanto en el norte, como en el sur. Además, se superpone o agrega, como fenómeno particular latinoamericano, el mestizaje entre portugués y castellano, entre inglés y castellano. Como lenguas funcionales de cada región de nuestro extenso país, podemos encontrar variedades de habla que contribuyen a revitalizar el plurilingüismo en la práctica, valorizándolo más allá de un posicionamiento teórico.

Es esta la perspectiva plurilingüe que asumimos frente a la enseñanza de lenguas, a la que debemos agregar una tercera dimensión: la de las lenguas funcionales socioculturales (sintetizando en este concepto los aportes teóricos de Coseriu y Rastier); es decir, cada grupo social, etario, de clase, de profesión, realiza una lengua funcional o dicho de otra manera, cada comunidad humana en relación con cada actividad que realiza usa una lengua funcional dada una lengua histórica.

Concebir el plurilingüismo como uso de varias lenguas y como reconocimiento de las diversas lenguas mestizas, así como las lenguas sociohistóricas que se hablan en cada comunidad, constituye un posicionamiento teórico e ideológico que consideramos beneficioso como docentes e investigadores en didáctica de las lenguas. 
En consecuencia, la idea de una lengua franca -a la manera de la propuesta de Comenio para el latín en la Europa del siglo XV-, que para algunos sería el inglés de hoy, no se sostiene conceptualmente desde una perspectiva plurilingüe. El inglés, en esta perspectiva, sería una lengua de servicio, no una lengua de comunicación, de cultura.

Desde este posicionamiento epistemológico y didáctico, la relación entre la actividad de un grupo social y la lengua funcional en uso tiene, además, el soporte teórico respecto de la constitución necesariamente dialogal del enunciado que aportara Voloshinov (2009). Es este autor, quien basándose en el estudio del diálogo de Jakubinskij (su maestro), asume y desarrolla el carácter dialogal del habla y acuña la noción de género de la palabra o del habla, concepto que revoluciona e interpela las ciencias sociales a fines del siglo XX.

Esta articulación necesaria entre la lengua funcional (en uso) y el carácter dialogal del enunciado, constitutivo de los géneros de habla como formatos de decir según la actividad extra verbal o extra lenguajera, es un punto de partida epistemológico que modifica el objeto de enseñanza de las lenguas: en este marco, la interacción es el eje conceptual que orienta la enseñanza de cualquier lengua funcional, ya que sin interlocutores no existe lengua viva.

En la década de 1990, Bronckart y Schneuwly (1991/1996), desde el interaccionismo sociodiscursivo, en Ginebra, desarrollaron el concepto de género textual, basándose en las nociones voloshinovianas de enunciado y género.

En consecuencia, desde esta perspectiva, uno de los desafíos que tenemos en América Latina en el contexto de la educación básica obligatoria (en la enseñanza de lenguas) es reconceptualizar el concepto de diálogo en el aula frente al plurilingüismo en sus diversas manifestaciones culturales actuales.

El plurilingüismo del aula se manifiesta en lenguas funcionales, como detallamos a continuación: en la lengua cultural docente, la lengua técnica docente, la lengua cotidiana entre estudiantes, la lengua intercultural entre docente-niños/adolescentes/jóvenes. Si delimitamos todas esas lenguas funcionales, podremos elegir -conscientemente- cómo comunicarnos en el aula. En síntesis, se trata de una tarea necesaria para la docencia en la actualidad: autorreflexionar sobre la lengua funcional en uso en cada contexto socio-discursivo.

\section{El aporte del análisis de habla dialogal de Jakubinskij}

El concepto de habla dialogal de Jakubinskij (2018) surge de las temáticas que -antes y después de la revolución de 1917 - interesaban a los lingüistas rusos sobre el habla cotidiana y el habla poética, la oralidad y el diálogo, la escritura y el monólogo. Todos estos conceptos estuvieron vigentes desde Baudoin de Courtenay (1845-1929), con reminiscencias precisas de Humboldt en los fundamentos.

Según Ivanova (2010), la concepción del carácter comunicacional de la lengua y el lenguaje humano de Baudoin de Courtenay es la que circuló entre lingüistas, filósofos y psicólogos de la época posrevolucionaria rusa, entre ellos, Vygotski, Voloshinov, Luria, Leontiev, Medvedev y el mismo Jakubinskij.

El concepto de habla, que proviene del concepto de actividad de lenguaje transmitido por Baudoin de Courtenay, profesor de Jakubinskij, es un aporte a la concepción de la funcionalidad ligada al concepto de lenguaje y a la lingüistica desde un enfoque psicosocial, en el sentido desarrollado por Humboldt respecto de la diversidad funcional.

Cabe destacar que el carácter de lo social como determinante está presente en los autores rusos y, si bien Jakubinskij parte de un análisis funcional, no es la "funcionalidad" de Jakobson -conocida en Occidente y ligada al propósito o finalidad del lenguaje en abstracto-, sino la diversidad funcional humboldtiana, que distingue "diversidad de formas" como variables de habla, por lo que esta diversidad funcional fue observada en diálogos reales. 
Jakubinskij le prestó atención a la fonación antes de que la sociolingüística surgiera como enfoque disciplinar. La articulación entre lo verbal y lo extraverbal es un concepto clave para entender la posición epistemológica de este lingüista ante el fenómeno del diálogo, diferenciándolo del monólogo:

Sin embargo, en nuestro caso, al tomar como punto de partida la distinción de las formas de habla, establecemos un puente entre los factores extralingüísticos y los fenómenos verbales y, entonces, tenemos, así, la posibilidad de hablar, por ejemplo, de la distinción de los medios de información en tal o tal variante, o bien de oponer directamente monólogo y diálogo como fenómenos verbales (Jakubinskij, 2018, p. 36).

Para Jakubinskij es el diálogo lo que contribuye a desarrollar la "automaticidad del habla". Esta automaticidad condiciona la variabilidad del habla dialogal por la presencia del interlocutor; es decir, la "percepción y la comprensión del otro” como elemento de retención. Identifica los cambios que produce el habla automática, que son: la aceleración, la reducción y la simplificación y, como puede verse, tiene una base empírica, cuyas observaciones son fonéticas.

En este sentido es que hace una crítica a la lingüística de su época por no estudiar la diversidad funcional del habla en toda su extensión, y propone estudiar diálogos reales como fenómenos particulares del habla. En el diálogo se reduce nuestra atención en la doble tarea de percibir y comprender el habla del interlocutor y de preparar (temática y verbalmente) la respuesta en el intervalo de dos réplicas. La estrechez de conciencia entre dos réplicas es una característica del diálogo, a diferencia del habla del monólogo, donde no hay coincidencia entre esos dos momentos.

En consecuencia, para Jakubinskij la diversidad funcional debe analizarse en diálogos reales en contraposición con monólogos (orales o escritos). El proceso de hablar es observado y definido como automatismo verbal (reacción natural espontánea de la réplica), por lo que el diálogo como fenómeno cultural está cercano a lo biológico ("psicofisiológico", dirá él), es un "fenómeno de la naturaleza”. Distingue: a) lo inacabado del diálogo (cada réplica supone una contrarréplica) y b) las interrupciones como necesarias en la interacción, ambas características señaladas entre otras, a partir de la observación empírica.

Esta definición del objeto de estudio de Jakubinskij, encuentra su base en las distinciones funcionales de Humboldt (1991, p.56) entre lenguaje hablado, lenguaje poético, el lógico-científico, del orador, todas distinciones de uso que, en alguna medida, coinciden con el germen del concepto de "género de la palabra" de Valentin Voloshinov (2009), alumno de Jakubinskij en su estudios de doctorado entre 1923 y 1926.

Hay, asimismo, otra influencia decisiva en los autores rusos de esta década, que es la del filósofo-psicólogo norteamericano William James (1842-1910), quien abordara el aspecto emocional del lenguaje y la explicación de la percepción como fenómeno. En particular, James abordó lo global y lo local como dimensiones del diálogo y éste como acción voluntaria simple, todos conceptos que tomará Jakubinskij directamente, citándolo a James en diversos trabajos.

Este tipo de influencias pone de manifiesto que los lingüistas y psicólogos rusos de la década del veinte y del treinta estaban en contacto con la producción intelectual occidental, situación que no se produjo a la inversa.

Tanto el lingüista Jakubinskij como el psicólogo Vygotski (2009) conocían y participaban de las discusiones de su época sobre el papel de las emociones en la comunicación y en el desarrollo humano.

Ivanova (2003/2010) sostiene que los conceptos de percepción y apercepción que Jakubinskij utilizó para su análisis del diálogo provienen de los desarrollos teóricos de William James y le sirvieron para explicar los aspectos rítmicos del diálogo. Ellos fueron denominados "clisés o estereotipos", pues reducen considerablemente la cantidad de palabras, las que pasan a un segundo plano por la simultaneidad de los dos procesos: comprensión y respuesta. 
La importancia del carácter aperceptivo de la percepción conduce a crear "estereotipos de la vida cotidiana" (suposiciones) y, en consecuencia, "estereotipos del habla" que son "determinantes de la percepción del habla". Se trata de la cotidianeidad como el medio ambiente cotidiano, así lo señala el autor. El proceso de hablar se apoya de tal modo en el medio ambiente que las interacciones verbales pasan a segundo plano en la percepción: "en la comunicación verbal dialógica no mediatizada la percepción de la entonación, visual, auditiva, etc. disminuirá la importancia de las palabras en cuanto tales". (Jakubinskij 2018, p.49).

Para explicar este concepto Jakubinskij pone el ejemplo de las frases hechas en la enseñanza de lenguas extranjeras, que son "sintácticamente complejas como estereotipos". La frase estereotipada habitual no se piensa sintagmáticamente, es sustantiva, la forman elementos habituales. En cambio, las actividades automáticas secundarias son actividades conscientes por repetición, lo que sería el caso de las repeticiones en otra lengua. En este sentido, la noción de estereotipo o clisé es interesante y muy actual para analizar la interacción como proceso de comunicación en un contexto determinado, ya que se trata de analizar la forma vivida como "forma percibida", que incluye la entonación, y la forma compartida, que se define a través del carácter social de las formas.

Es importante destacar que a Jakubinskij le interesó el habla dialogal como fenómeno y como forma no mediatizada, que comparó con otras formas mediatizadas como la escritura y el monólogo. Lo que observó fue la percepción auditiva y visual, por lo que abordó el diálogo como proceso de hablar. Delimitó con precisión descriptiva y funcional el automatismo verbal en la réplica, en el interlocutor, en el papel de la retención en tanto percepción y comprensión, aspectos que anticiparon el análisis conversacional de Sacks (1974) y el cognitivismo actual (Paas, Renkl y Sweller, 2004).

Según Bertau (2008), la forma del análisis de los lingüistas y psicólogos rusos es compleja y abarcadora, ya que comprende tanto la actividad del lenguaje como el pensamiento y la conciencia, los géneros, la dialogicidad y lo predicativo del habla interior, desde una perspectiva de forma vivida, que no es la forma abstracta mayormente divulgada entre los lingüistas.

Como lo señalo más arriba, cabe destacar que es la "diversidad funcional" humboldtiana la que distingue "diversidad de formas", observada por Jakubinskij en diálogos reales, prestando atención a la fonación antes de que la sociolingüística surgiera como enfoque disciplinar. Es por eso que este estudio se anticipó casi medio siglo.

A modo de síntesis, observamos que la tradición rusa heredada del siglo XIX, con un enfoque lingüístico formal y psicológico, fue la que a partir de la fonética y de la actividad de lenguaje delimitó el diálogo en un análisis que aún hoy mantiene vigencia y es lo que nos permite esbozar perspectivas nuevas en la investigación educativa.

Por otra parte, en coincidencia con el concepto saussuriano de signo, en un artículo previo de Jakubinskij, publicado en 1916 (traducido al francés como "Sur les sons de la langue versifiée"), el ruso comparó la lengua práctica y la lengua poética, donde, además, sostuvo el principio semántico de la lengua frente al fonológico, un aspecto aún no registrado en la lingüística de su época:

Dans la pensée langagière pratique, le locuteur no concentre pas son attention sur les sons: les sons n'entrent pas dans le champ clair de sa conscience et ne possèdent pas de valeur indépendante, car ils ne servent qu'a communiquer. Dans ce cas, l'aspect sémantique des mots joue un rôle plus important que l'aspect sonore. Les détails de la prononciation entrent dans la conscience seulement pour la distinction du sens (citado por Ivanova, 2009, p.117). 
Esta distinción entre el aspecto semántico de la lengua práctica y el papel de los sonidos en la comunicación como vehículos del sentido es una observación que supera lo que enfocaban los lingüistas en la época.

En la actualidad resulta auspicioso aún que los conceptos de Jakubinskij echen luz sobre la problemática de la comunicación humana en nuestro campo de investigación, que es la didáctica de las lenguas.

\section{Análisis del diálogo en la escuela}

En el aire del tiempo de la Rusia revolucionaria, los estudios interdisciplinarios que se realizaban en el Instituto de la Palabra Viviente (Institut Zivoe Slovo) aportaron e institucionalizaron la idea de síntesis entre las ciencias y las artes frente a un nuevo objeto de investigación que era la palabra viviente. Al respecto sostiene Ivanova (2008): "El Instituto de la palabra viviente era un niño del pensamiento revolucionario y de la época de las grandes esperanzas" (p.149, traducción nuestra). Funcionó en Petrogrado entre 1918 y 1924. Jakubinskij fue uno de sus fundadores y, aunque no buscó analizar en profundidad el diálogo, según afirma en su texto, su trabajo fue precursor en los estudios del lenguaje al situar el objeto en las fronteras disciplinares, algo que en la época era una novedad.

En nuestra investigación partimos del concepto ruso de actividad de lenguaje o actividad verbal, actividad lenguajera o actividad lenguajeante -todas posibles traducciones para ampliar la semanticidad del término-, adoptado por otros autores referenciales, como Vygotski y Voloshinov y, en particular en la actualidad, por Bronckart (1997; 2001). Es esta la actividad consciente que nos diferencia de las otras especies vivas y que media todas las otras actividades humanas. En nuestra investigación nos interesa saber qué papel juega la automatización dentro de esta actividad y, en particular, cuál es la importancia de este aspecto (lo automático del diálogo) en clases de lengua de primario y de secundario.

Hemos analizado la coincidencia del concepto de lenguaje entre Vygotski, Leontiev y Voloshinov (Riestra, 2014) con enfoques específicos, como el desarrollo del lenguaje en el primero, la actividad de lenguaje en el segundo y el diálogo en el tercero. Todos consideran lo colectivo y lo social como determinante de lo individual, aunque con matices respecto del papel de la individualidad y la autorreflexión a través del lenguaje. Podemos decir que hay una congruencia epistemológica en la concepción de la actividad lenguajera entre estos tres autores rusos en los que nos basamos en nuestras investigaciones.

Actualmente, desde la perspectiva de Jakubinskij (2018), estamos investigando en aulas de primario y de secundario el habla dialogal entre profesoras y profesores con sus alumnos. La "lengua funcional” de Coseriu (1992) es otro concepto instrumental en nuestra investigación, cuya distinción tiene la finalidad de aportar a la práctica profesional docente en enseñanza de lenguas.

La metodología que desarrollamos apunta a elaborar instrumentos de evaluación para la práctica profesional docente, en función de la interacción discursivo-textual en el aula. En primer lugar, realizamos el análisis de las consignas de trabajo (Riestra, 2008); en segundo lugar, el análisis de las réplicas y a través de las mismas las masas aperceptivas de profesores y estudiantes en el aula. De este modo, abordamos dos dimensiones que, articuladas metodológicamente, nos permiten conocer mejor la situación plurilingüística en la escuela en los niveles primario y secundario.

El primer análisis es el de la consigna como acción de la actividad de lenguaje. Este tipo de análisis consiste en delimitar las tareas y las actividades propuestas a través de las consignas a los estudiantes, con la finalidad de observar la relación entre lo que se les dice que deben hacer y lo que efectivamente hacen. De este modo evaluamos el desarrollo de las capacidades discursivo-textuales (orales o escritas) de los estudiantes, en la medida en que se puede cotejar lo 
propuesto a través de las consignas con lo realizado por los destinatarios de las consignas. Asimismo, este análisis nos permite evaluar la pertinencia de las consignas formuladas y reformuladas durante la clase.

Entendemos que las consignas contienen una intencionalidad dialogal, pero, además, un fuerte sesgo monologal (lo que se espera que se entienda y se haga) por estar planificadas por escrito en un contexto previo al de la clase, que podemos resumir de este modo en la Tabla 1:

\begin{tabular}{|l|}
\hline Análisis de consignas \\
\hline El docente dice para que los alumnos hagan \\
\hline Es un espacio verbal y mental planificado (Autor) \\
\hline $\begin{array}{l}\text { El docente se sitúa en el proceso de desarrollo potencial del interlocutor-alumno } \\
\text { para formular verbalmente la tarea que éste deberá realizar }\end{array}$ \\
\hline
\end{tabular}

Tabla 1: Consignas. Fuente: elaboración propia.

Por otra parte, en el método de análisis del diálogo de Jakubinslij (2018) el proceso de hablar es referido y descripto como "automatismo verbal", la reacción natural espontánea de la réplica. En investigaciones anteriores, al analizar las consignas el automatismo del habla dialogal aparecía como fondo, contexto o medio ambiente de la clase, sin que encontráramos metodológicamente el enfoque que lo integrara o, en todo caso, nos referíamos a la clase como texto global, en sentido amplio y a las consignas como textos en un sentido más restringido (Riestra, 2015; 2018).

En el análisis de Jakubinskij, al diferenciar diálogo de monólogo y considerar como natural el primero y artificial el segundo, encontramos una posibilidad de articular metodológicamente los dos niveles de análisis: a) los textos de las consignas y sus efectos como textos considerados monologales, lo que en cierta medida modifica nuestra conceptualización o, mejor dicho, la complejiza; b) el análisis de las réplicas de la clase como análisis de las masas aperceptivas (grabación y transcripción de las clases).

Como se ha indicado antes, Jakubinskij analiza el habla mediante las réplicas (término que él mismo creara especialmente en ruso: replicirovanie) como constitutivas del diálogo, basándose en los planteos de Scherba (1926). Es en las réplicas donde podemos observar cómo se produce la apercepción coincidente o divergente entre profesores y alumnos. Se trata de atender a los "prejuicios" o suposiciones respecto del otro, algo que sucede en todos los contextos y, en particular, en el aula por la asimetría de los roles entre los interlocutores. De hecho, muchas veces encontramos la no coincidencia entre las apercepciones de docentes y los alumnos en lo que hace a las expectativas docentes o a los malentendidos con los alumnos, en la medida en que los docentes no registran que los alumnos no entienden algunas nociones técnicas expresadas.

También utilizamos la noción de "estereotipo o clisé" como categoría. Es esta una noción que comprende tanto lo gestual, lo supuesto, como las frases hechas en cada contexto; por lo tanto, se trata de una categoría que nos permite relacionar texto y contexto, desde la perspectiva interaccionista. 
En la Tabla 2 sintetizamos las categorías que estamos utilizando para analizar el diálogo:

\begin{tabular}{|l|l|}
\hline Habla dialogal automática & Masas aperceptivas de alumnos/profesores \\
\hline $\begin{array}{l}\text { Se entiende según los supuestos (apercepciones) } \\
\text { No coincidencia entre profesor y alumnos /a veces } \\
\text { hay coincidencia }\end{array}$ \\
\hline $\begin{array}{l}\text { Las nociones técnicas y el sentido común se } \\
\text { intercalan en las réplicas. }\end{array}$ & $\begin{array}{l}\text { Espacio a construir desde el profesor para que las } \\
\text { masas aperceptivas coincidan }\end{array}$ \\
\hline
\end{tabular}

Tabla 2: Categorías de análisis del diálogo. Fuente: elaboración propia.

$\mathrm{Al}$ introducir la percepción en la descripción del habla dialogal, se observa el proceso de la interiorización de la palabra del otro y el efecto que produce la réplica del otro. Por lo tanto, uno de los aspectos no verbales o complementarios de la verbalización dialogal, que Jakubinskij denomina como "la percepción y la apercepción”, se señala en relación con la comprensión y la continuidad de la comunicación.

Desde una perspectiva didáctica, el sentido compartido o no de los enunciados en el aula es un proceso verbal al que debería prestarse más atención como proceso comunicacional. El habla dialogal del aula como problemática comunicacional actual es lo que nos orienta en la elaboración de un instrumento de autoevaluación del trabajo docente (objeto de síntesis metodológica posterior), en la medida en que el análisis del diálogo muestra, a través de las réplicas, los enunciados del propio docente y la relación con los enunciados de los alumnos.

También observamos y registramos lo inacabado del habla dialogal (a diferencia del monólogo), aspecto que retomará Voloshinov (2009) al incorporar la noción de entimema. Dicho de otro modo, los sobreentendidos, los malentendidos, lo incomprendido pueden ser observados en los registros auditivos de la interacción.

Nuestro análisis se complementa con las nociones de Coseriu (1992) respecto del habla y su funcionalidad al delimitar y explicar el concepto de "lengua funcional", que notamos muy próximo a lo que Jakubinskij identifica como las variables del habla, desde la diversidad funcional. El concepto de lengua funcional es relevante para la investigación porque nos permite relacionar texto y contexto, una relación que, por lo general, en las formalizaciones de los análisis didácticos tiende a diluirse. En consecuencia, la concepción plurilingüe del lenguaje humano aporta en un análisis didáctico en la actualidad, en particular como herramienta para estudiar las formas que son denominadas "lenguajes inclusivos", las que podemos considerar como variables de habla o lenguas funcionales.

Es en esta dirección que analizamos los enunciados como signos lingüísticos que vehiculizan masas aperceptivas (coincidentes o no) a través de los cuatro tipos discursivos (Bronckart, 1997; 2001) vehiculizados en las lenguas funcionales (de docentes y de alumnos). Son los que Bronckart identifica como cuatro formatos psicolenguajeros del contar y el exponer que se producen en diversas lenguas estudiadas: el relato interactivo (el autor se implica), la narración (el autor no se implica), el discurso interactivo (el autor se implica), y el discurso teórico (el autor no se implica). Se trata de formatos automatizados, identificables en un análisis textual a través de formas lingüísticas verbales y pronominales. La relación entre el diálogo, las lenguas y los formatos discursivos pueden verse en la siguiente tabla: 


\begin{tabular}{|l|l|}
\hline Diálogo profesor-alumno & Tipos discursivos y lenguas funcionales \\
\hline $\begin{array}{l}\text { Réplicas de profesores y alumnos. } \\
\text { Signos lingüísticos (Saussure) en textos de la clase. }\end{array}$ & $\begin{array}{l}\text { Formatos lenguajeros del contar y el exponer autó- } \\
\text { nomo e implicado) } \\
\text { Son las operaciones psico-verbales (Bronckart) de } \\
\text { profesores y alumnos (aparecen en las consignas). }\end{array}$ \\
\hline $\begin{array}{l}\text { Masas aperceptivas coincidentes o divergentes } \\
\text { (Jakubinskij) }\end{array}$ & $\begin{array}{l}\text { Lengua funcional (Coseriu) o } \\
\text { Dialecto social (Jakubinskij) de los profesores } \\
\text { y de los alumnos alumnos }\end{array}$ \\
\hline
\end{tabular}

Tabla 3: Lenguas funcionales y tipos discursivos en el diálogo del aula. Fuente: Elaboración propia.

\section{La cuestión del diálogo y el fenómeno del plurilingüismo}

El fondo ideológico-teórico del marxismo tuvo, sin dudas, una influencia relevante en la época de la revolución rusa en la que se hicieron los primeros estudios del diálogo como forma de la comunicación humana, pero más allá de esta influencia del aire del tiempo, es necesario conocer y entender que existió una tradición cultural rusa que hasta hoy atraviesa las concepciones de los lingüistas, psicólogos, filósofos, es decir, de los estudios de las ciencias humanas-sociales. La concepción del lenguaje como actividad tiene raíces profundas en la perspectiva de la percepción de los fenómenos y su análisis en este plano, como empirias, no como ideas acerca de los datos empíricos elegidos. En esta perspectiva, según Seriot (2011), estudioso de lenguas eslavas, las nociones como "discurso" y "sujeto" carecen de entidad en la lengua rusa y, en consecuencia, al no existir, no tienen ningún significado.

Además, Sériot (2008) sostiene que las ideas provenientes de Rusia fueron integradas en los debates existentes en psicología, teoría literaria, lingüística, sociología, etc., sin prestar atención a su contexto de producción y recepción.

Lo que podría plantearse como una cuestión de traducción, para este eslavista de origen francés, no es simplemente eso, ya que agrega lo siguiente:

Se prestó atención a grandes nombres, pero se ignoró la riqueza considerable de Rusia como laboratorio de las ciencias humanas y sociales [...] La cultura rusa, si bien no es la alteridad de China o Japón, tampoco es la misma cosa que Europa occidental. Digamos, un otro nosotros mismos, otra manera de ser europeos (Sériot, 2008, p. 2, traducción nuestra).

Por lo tanto, la cuestión de la traducción no es menor frente a la problemática del plurilingüismo. En consonancia con este señalamiento, hemos registrado en las traducciones del ruso al francés, al portugués, al inglés y al castellano, errores de significaciones, que en la mayoría de los casos conforman malentendidos teóricos como "sujeto discursivo", concepto inexistente, del mismo modo que el concepto de género discursivo, cuya interpretación occidentalizada no coincide con el significado y el sentido de los textos rusos, todo esto avalado por estudios históricos y epistemológicos realizados últimamente en la Universidad de Lausanne. Estas investigaciones lingüísticas que mencionamos constituyen un aporte para situarnos en una perspectiva plurilingüe del lenguaje humano, que es una perspectiva basada no solamente en la diversidad de lenguas históricas, sino en la diversidad funcional de las lenguas. Para aclarar el concepto de plurilingüismo al que nos referimos, en un artículo de 1924, Scherba (1926), otro lin- 
güista ruso contemporáneo de Jakubinskij, coloca la cuestión de las "lenguas mixtas" como diferentes dialectos que cada persona habla en diversos medios, por lo que para él todos seríamos "polilingües". De esta concepción se deriva la influencia recíproca de las lenguas y Scherba alude específicamente a dos procesos que a menudo se entrecruzan: el préstamo y la mezcla de lenguas. Sostiene que el primero se basa en el bilingüismo (coexistencia de dos lenguas en un mismo individuo) y el segundo se basa en una lengua mixta de dos términos. Parece que el desarrollo del bilingüismo o de la lengua mixta de dos términos depende: 1) de un modo de aprendizaje de la lengua segunda y 2) de la delimitación de los dominios de empleo de las dos lenguas.

La mezcla de lenguas no implica, necesariamente, la pérdida del sentimiento de continuidad de una lengua dada. Pareciera que ciertos cambios provienen sobre todo de la mezcla de lenguas, como cambios de la "forma interior", pero en la mayor parte de los casos no se sabe sobre el proceso por el que se reúnen elementos heterogéneos de una lengua dada. Estas problemáticas, propuestas para ser estudiadas a principios del siglo pasado, aún hoy continúan interrogándonos, tanto desde el aula de enseñanza de la lengua primera como de las lenguas segundas. Sigue interpelándonos una problemática de las lenguas como fenómeno de la comunicación humana.

En nuestra investigación, para entender la semiosis como proceso de la interacción verbal, revisitamos al Saussure (2004) de sus escritos inéditos encontrados como notas en 1996, quien da una respuesta revolucionaria a la problemática del cambio permanente de las lenguas, de ruptura con la hipótesis de derivación de las lenguas.

En continuidad con trabajos anteriores anteriores (Riestra, 2008; 2010; 2015; 2017; 2018), en el presente ensayo se busca aportar en la dirección de la didactización de los conceptos gramaticales. Y, a la vez, en la comunicación con los alumnos, teniendo en cuenta que las lenguas funcionales de los alumnos y las apercepciones que se producen en el aula no son las mismas que las de los docentes.

Nuestro marco teórico metodológico se organiza desde la articulación conceptual del mecanismo del lenguaje (lengua interna y lengua externa) de Saussure; las lenguas históricas y funcionales, según Coseriu; y las lenguas mixtas, de acuerdo con los estudios de los lingüistas rusos de la década de 1920-30. Por otra parte, el análisis del diálogo de Jakubinskij nos permite entender cómo se producen en el aula las dos modalidades atribuidas al habla dialogal a través de las réplicas que, por necesidad, deben interiorizarse para: a) retener y comprender, a la vez que para b) suceder en el habla o interrumpir.

En síntesis, buscamos que este tipo de análisis tenga una utilidad autoevaluativa de los enunciados producidos en la interacción verbal del aula. En un primer momento, como docentes, podemos evaluar la formulación de nuestras consignas en los contextos reales de enseñanza, analizando, además, las tareas realizadas por los alumnos. En un segundo momento, con el análisis del diálogo podemos evaluar el efecto de nuestras consignas y sus reformulaciones, así como las explicaciones discursivo-textuales dadas a los alumnos y los conceptos técnicos instrumentados. En definitiva, investigar la enseñanza de las lenguas no puede prescindir del análisis de la comunicación durante el proceso de formalización del objeto de estudio. Si bien se enseña el uso de una lengua, sea esta materna o primera, segunda o técnica, siempre estaremos trabajando en la conformación y el desarrollo del instrumento de la comunicación humana, que es el lenguaje mediado por las lenguas. 


\section{Bibliografía}

Bertau, M.C. (2008). Pour une notion de forme linguistique comme forme vécue. Une approche avec Jakubinskij, Volosinov et Vygotski in Langage et pensée: Union sovietique années 1920-1930. Lausanne: UNIL.

Bronckart, Jean-Paul (1997). Activité langagière, textes et discours. Pour un interactionisme socio-discursif. ParisGenève : Delachaux et Niestlé

Bronckart,J.-P.(2001).Entenderse para actuar y actuar para entenderse. (S'entendre pour agir et agir pour s'entendre), Raisons Educatives 4. Théories de l'action et interventions formatives.

Coseriu, E. (1992). Competencia lingüistica. Madrid: Gredos.

Humboldt, W. (1991) Escritos sobre el lenguaje. Barcelona: Ed. Península.

Ivanova, I. (2010). El diálogo en la lingüística soviética de los años 1920-1930. en Riestra, D. Saussure, Voloshinov y Bajtin revisitados. Estudios históricos y epistemológicos. Buenos Aires: Miño\& Dávila.

Ivanova, I. (2008). Le rôle de l'Institut Zivogo Slova (Petrograd) dans la culture russe du debut du XXe siècle en Langage et pensée:Union Soviétique années 1920-1930. Lausanne: UNIL

Ivanova, I. (2012). Lev Jakubinskij, une linguistique de la parole. Lausanne: Lambert Lucas

Jakubinskij, L. (2015). Sobre a fala dialogal. Sao Paulo: Parábola.

Jakubinskij, L. (2018). Sobre el habla dialogal. Viedma: Editorial UNRN.

Paas, F; Renkl, A. \& Sweller, J. (2004). Cognitive Load Theory: Instructional Implications of the Interaction between Information Structures and Cognitive Architecture. Instructional Science 32, pp. 1-8.

Rastier, F. (2012). Aprender en Terceras jornadas internacionales de investigación y prácticas en didáctica de las lenguas y las literaturas. Bariloche, 27 de octubre de 2012. Ed. UNRN

Riestra, D. (2008). Las consignas de enseñanzas de la lengua: Un análisis desde el interaccionsimo socio-discursivo. Buenos Aires: Miño\& Dávila

Riestra, D., ed. (2010). Saussure, Volóshinov y Bajtín revisitados. Estudios históricos y epistemológicos. Buenos Aires: Miño y Dávila.

Riestra, D. (2014). Saussure, Vygotski and Voloshinov: the linguistic sign as an epistemological issue. En The Linguist Society of St. Petersburg (Ed.). Language and language behavior. St Petersburg: St Petersburg State University-Faculty of Philology, pp. 140-147.

Riestra, D. (2015). La vigencia de Saussure y la enseñanza de las lenguas. Eutomia 16 (1), pp. 259-274.

Riestra, D. (2017). La concepción del diálogo de los rusos desde la perspectiva interaccionista socio-discursiva. Veredas-Interacionismo sociodiscursivo 1, pp. 11-29.

Riestra, D. (2018). La interacción en la clase de lengua y literatura: análisis desde el diálogo. Revista de la Escuela de Ciencias de la Educación, año 14, nro. 13, vol. 1, enero a junio, pp. 167-179. 
Sacks, H.; Shegloff, E; Jefferson, G. (1974). A simplest systematic for the organization of turn- talking for conversation. Language, Vol 50, n 4, part 1.(dec. 1974) pp 696-735.

Ščerba, L. (1926). Sur la notion de mélange des langues, Яфетический Сборник-Recueil Japhétique, IV: Проблемьь, 1926, АенинграА : Яфетический Институт Академии Наук СССР, p. 1-19.

Saussure, F. (2004). Escritos sobre lingüistica general. Barcelona: Gedisa.

Sériot, P. (2008). Langage et pensé:Union Soviiétique annés 1920-1930. Cahiers de L’ILSL, n²4.

Lausanne: UNIL

Sériot, P. (2011). Russie, linguistique et philosophie. de L'ILSL, n²9. Lausanne: UNIL

Voloshinov, V. (2009). Marxismo y filosofia del lenguaje. Buenos Aires: Godot

Vygotski, L. (2009). Pensamiento y habla. Buenos Aires: Colihue. 